\title{
Automatic Extraction of Subcategorization from Corpora
}

\author{
Ted Briscoe \\ Computer Laboratory \\ University of Cambridge \\ Pembroke Street, Cambridge CB2 3QG, UK \\ ejb@cl.cam.ac.uk \\ John Carroll \\ Cognitive and Computing Sciences \\ University of Sussex \\ Brighton BN1 9QH, UK \\ john.carroll@cogs.susx.ac.uk
}

\begin{abstract}
We describe a novel technique and implemented system for constructing a subcategorization dictionary from textual corpora. Each dictionary entry encodes the relative frequency of occurrence of a comprehensive set of subcategorization classes for English. An initial experiment, on a sample of 14 verbs which exhibit multiple complementation patterns, demonstrates that the technique achieves accuracy comparable to previous approaches, which are all limited to a highly restricted set of subcategorization classes. We also demonstrate that a subcategorization dictionary built with the system improves the accuracy of a parser by an appreciable amount ${ }^{1}$.
\end{abstract}

\section{Motivation}

Predicate subcategorization is a key component of a lexical entry, because most, if not all, recent syntactic theories 'project' syntactic structure from the lexicon. Therefore, a wide-coverage parser utilizing such a lexicalist grammar must have access to an accurate and comprehensive dictionary encoding (at a minimum) the number and category of a predicate's arguments and ideally also information about control with predicative arguments, semantic selection preferences on arguments, and so forth, to allow the recovery of the correct predicate-argument structure. If the parser uses statistical techniques to rank analyses, it is also critical that the dictionary encode the relative frequency of distinct subcategorization classes for each predicate.

\footnotetext{
${ }^{1}$ This work was supported by UK DTI/SALT project $41 / 5808$ 'Integrated Language Database', CEC Telematics Applications Programme project LE1-2111 'SPARKLE: Shallow PARsing and Knowledge extraction for Language Engineering', and by SERC/EPSRC Advanced Fellowships to both authors. We would like to thank the COMLEX Syntax development team for allowing us access to pre-release data (for an early experiment), and for useful feedback.
}

Several substantial machine-readable subcategorization dictionaries exist for English, either built largely automatically from machine-readable versions of conventional learners' dictionaries, or manually by (computational) linguists (e.g. the Alvey NL Tools (ANLT) dictionary, Boguraev et al. (1987); the COMLEX Syntax dictionary, Grishman et al. (1994)). Unfortunately, neither approach can yield a genuinely accurate or comprehensive computational lexicon, because both rest ultimately on the manual efforts of lexicographers / linguists and are, therefore, prone to errors of omission and commission which are hard or impossible to detect automatically (e.g. Boguraev \& Briscoe, 1989; see also section 3.1 below for an example). Furthermore, manual encoding is labour intensive and, therefore, it is costly to extend it to neologisms, information not currently encoded (such as relative frequency of different subcategorizations), or other (sub)languages. These problems are compounded by the fact that predicate subcategorization is closely associated to lexical sense and the senses of a word change between corpora, sublanguages and/or subject domains (Jensen, 1991).

In a recent experiment with a wide-coverage parsing system utilizing a lexicalist grammatical framework, Briscoe \& Carroll (1993) observed that half of parse failures on unseen test data were caused by inaccurate subcategorization information in the ANLT dictionary. The close connection between sense and subcategorization and between subject domain and sense makes it likely that a fully accurate 'static' subcategorization dictionary of a language is unattainable in any case. Moreover, although Schabes (1992) and others have proposed 'lexicalized' probabilistic grammars to improve the accuracy of parse ranking, no wide-coverage parser has yet been constructed incorporating probabilities of different subcategorizations for individual predicates, because of the problems of accurately estimating them.

These problems suggest that automatic construction or updating of subcategorization dictionaries from textual corpora is a more promising avenue to pursue. Preliminary experiments acquiring a few 
verbal subcategorization classes have been reported by Brent (1991, 1993), Manning (1993), and Ushioda et al. (1993). In these experiments the maximum number of distinct subcategorization classes recognized is sixteen, and only Ushioda et al. attempt to derive relative subcategorization frequency for individual predicates.

We describe a new system capable of distinguishing 160 verbal subcategorization classes-a superset of those found in the ANLT and COMLEX Syntax dictionaries. The classes also incorporate information about control of predicative arguments and alternations such as particle movement and extraposition. We report an initial experiment which demonstrates that this system is capable of acquiring the subcategorization classes of verbs and the relative frequencies of these classes with comparable accuracy to the less ambitious extant systems. We achieve this performance by exploiting a more sophisticated robust statistical parser which yields complete though 'shallow' parses, a more comprehensive subcategorization class classifier, and a priori estimates of the probability of membership of these classes. We also describe a small-scale experiment which demonstrates that subcategorization class frequency information for individual verbs can be used to improve parsing accuracy.

\section{Description of the System}

\subsection{Overview}

The system consists of the following six components which are applied in sequence to sentences containing a specific predicate in order to retrieve a set of subcategorization classes for that predicate:

1. A tagger, a first-order HMM part-of-speech ( $\mathrm{PoS}$ ) and punctuation tag disambiguator, is used to assign and rank tags for each word and punctuation token in sequences of sentences (Elworthy, 1994).

2. A lemmatizer is used to replace word-tag pairs with lemma-tag pairs, where a lemma is the morphological base or dictionary headword form appropriate for the word, given the PoS assignment made by the tagger. We use an enhanced version of the GATE project stemmer (Cunningham et al., 1995).

3. A probabilistic $\mathbf{L R}$ parser, trained on a treebank, returns ranked analyses (Briscoe \& Carroll, 1993; Carroll, 1993, 1994), using a grammar written in a feature-based unification grammar formalism which assigns 'shallow' phrase structure analyses to tag networks (or 'lattices') returned by the tagger (Briscoe \& Carroll, 1994, 1995; Carroll \& Briscoe, 1996).

4. A patternset extractor which extracts subcategorization patterns, including the syntactic categories and head lemmas of constituents, from sentence subanalyses which begin/end at the boundaries of (specified) predicates.

5. A pattern classifier which assigns patterns in patternsets to subcategorization classes or rejects patterns as unclassifiable on the basis of the feature values of syntactic categories and the head lemmas in each pattern.

6. A patternsets evaluator which evaluates sets of patternsets gathered for a (single) predicate, constructing putative subcategorization entries and filtering the latter on the basis of their reliability and likelihood.

For example, building entries for attribute, and given that one of the sentences in our data was (1a), the tagger and lemmatizer return (1b).

(1) a $\mathrm{He}$ attributed his failure, he said, to no<blank>one buying his books.

b he_PPHS1 attribute_VVD his_APP\$ failure_NN1,-, he_PPHS1 say_VVD ,, to_II $n o<b l a n k>0 n e_{-} P N \quad$ buy_VVG his_APP\$ book NN2

(1b) is parsed successfully by the probabilistic LR parser, and the ranked analyses are returned. Then the patternset extractor locates the subanalyses containing attribute and constructs a patternset. The highest ranked analysis and pattern for this example are shown in Figure $1^{2}$. Patterns encode the value of the VSUBCAT feature from the VP rule and the head lemma(s) of each argument. In the case of PP (P2) arguments, the pattern also encodes the value of PSUBCAT from the PP rule and the head lemma(s) of its complement(s). In the next stage of processing, patterns are classified, in this case giving the subcategorization class corresponding to transitive plus PP with non-finite clausal complement.

The system could be applied to corpus data by first sorting sentences into groups containing instances of a specified predicate, but we use a different strategy since it is more efficient to tag, lemmatize and parse a corpus just once, extracting patternsets for all predicates in each sentence; then to classify the patterns in all patternsets; and finally, to sort and recombine patternsets into sets of patternsets, one set for each distinct predicate containing patternsets of just the patterns relevant to that predicate. The tagger, lemmatizer, grammar and parser have been described elsewhere (see previous references), so we provide only brief relevant details here, concentrating on the description of the components

\footnotetext{
${ }^{2}$ The analysis shows only category aliases rather than sets of feature-value pairs. Ta represents a text adjunct delimited by commas (Nunberg 1990; Briscoe \& Carroll, 1994). Tokens in the patternset are indexed by sequential position in the sentence so that two or more tokens of the same type can be kept distinct in patterns.
} 
(Tp

(V2 (N2 he_PPHS1)

(V1 (V0 attribute_VVD)

(N2 (DT his_APP\$)

(N1

(NO (NO failure_NN1)

( Ta $(\mathrm{Pu},-$,

(V2 (N2 he_PPHS1)

$($ V1 (V0 say_VVD) $))(P u,,))))$,

(P2

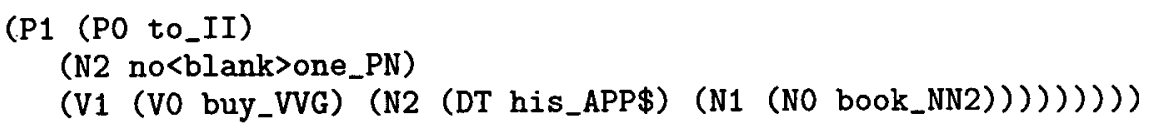

(P1 (PO to_II)

(N2 no<blank>one_PN)

$(V 1(V 0$ buy_VVG) (N2 (DT his_APP\$) $(N 1($ NO book_NN2)) )) )) )) ))

Figure 1: Highest-ranked analysis and patternset for (1b)
(1 (( ( he: 1 PPHS1))

(VSUBCAT NP_PP)

((attribute:6 VVD))

((failure:8 NN1))

((PSUBCAT SING)

( (to:9 II ))

( (no<blank>one:10 PN ))

((buy:11 VVG))))

. 1)) of the system the

The grammar consists of 455 phrase structure rule schemata in the format accepted by the parser (a syntactic variant of a Definite Clause Grammar with iterative (Kleene) operators). It is 'shallow' in that no atof which thetempt is made to fully analyse unbounded dependencies. However, the distinction between arguments and adjuncts is expressed, following X-bar theory (e.g. Jackendoff, 1977), by Chomsky-adjunction to maximal projections of adjuncts (XP $\rightarrow$ XP Adjunct) as opposed to 'government' of arguments (i.e. arguments are sisters within $\mathrm{X} 1$ projections; $\mathrm{X} 1 \rightarrow \mathrm{X} 0 \mathrm{Arg} 1 \ldots \mathrm{ArgN})$. Furthermore, all analyses are rooted (in $\mathrm{S}$ ) so the grammar assigns global, shallow and often 'spurious' analyses to many sentences. There are 29 distinct values for VSUBCAT and 10 for PSUBCAT; these are analysed in patterns along with specific closed-class head lemmas of arguments, such as it (dummy subjects), whether (wh-complements), and so forth, to classify patterns as evidence for one of the $160 \mathrm{sub}$ categorization classes. Each of these classes can be parameterized for specific predicates by, for example, different prepositions or particles. Currently, the coverage of this grammar-the proportion of sentences for which at least one analysis is found-is $79 \%$ when applied to the Susanne corpus (Sampson, 1995), a 138K word treebanked and balanced subset of the Brown corpus. Wide coverage is important since information is acquired only from successful parses. The combined throughput of the parsing components on a Sun UltraSparc 1/140 is around 50 words per CPU second.

\subsection{The Extractor, Classifier and Evaluator}

The extractor takes as input the ranked analyses from the probabilistic parser. It locates the subanalyses around the predicate, finding the constituents identified as complements inside each subanalysis, and the subject clause preceding it. Instances of passive constructions are recognized and treated specially. The extractor returns the predicate, the VSUBCAT value, and just the heads of the complements (except in the case of PPs, where it returns the PSUBCAT value, the preposition head, and the heads of the PP's complements).

The subcategorization classes recognized by the classifier were obtained by manually merging the classes exemplified in the COMLEX Syntax and ANLT dictionaries and adding around 30 classes found by manual inspection of unclassifiable patterns for corpus examples during development of the system. These consisted of some extra patterns for phrasal verbs with complex complementation and with flexible ordering of the preposition/particle, some for non-passivizable patterns with a surface direct object, and some for rarer combinations of governed preposition and complementizer combinations. The classifier filters out as unclassifiable around $15 \%$ of patterns found by the extractor when run on all the patternsets extracted from the Susanne corpus. This demonstrates the value of the classifier as a filter of spurious analyses, as well as providing both translation between extracted patterns and two existing subcategorization dictionaries and a definition of the target subcategorization dictionary.

The evaluator builds entries by taking the patterns for a given predicate built from successful parses and records the number of observations of each subcategorization class. Patterns provide several types of information which can be used to rank or select between patterns in the patternset for a given sentence exemplifying an instance of a predicate, such as the ranking of the parse from which it was extracted or the proportion of subanalyses supporting a specific pattern. Currently, we simply select the pattern supported by the highest ranked parse. However, we are experimenting with alternative approaches. The resulting set of putative classes for a predicate are filtered, following Brent (1993), 
by hypothesis testing on binomial frequency data.

Evaluating putative entries on binomial frequency data requires that we record the total number of patternsets $n$ for a given predicate, and the number of these patternsets containing a pattern supporting an entry for given class $m$. These figures are straightforwardly computed from the output of the classifier; however, we also require an estimate of the probability that a pattern for class $i$ will occur with a verb which is not a member of subcategorization class $i$. Brent proposes estimating these probabilities experimentally on the basis of the behaviour of the extractor. We estimate this probability more directly by first extracting the number of verbs which are members of each class in the ANLT dictionary (with intuitive estimates for the membership of the novel classes) and converting this to a probability of class membership by dividing by the total number of verbs in the dictionary; and secondly, by multiplying the complement of these probabilities by the probability of a pattern for class $i$, defined as the number of patterns for $i$ extracted from the Susanne corpus divided by the total number of patterns. So, $p(v-i)$, the probability of verb $v$ not of class $i$ occurring with a pattern for class $i$ is:

$p(v-i)=\left(1-\frac{\mid \text { anlt_verbs_in_class_i } i \mid}{\mid \text { anlt_verbs } \mid}\right) \frac{\mid \text { patterns_for_i } \mid}{\mid \text { patterns } \mid}$

The binomial distribution gives the probability of an event with probability $p$ happening exactly $m$ times out of $n$ attempts:

$$
P(m, n, p)=\frac{n !}{m !(n-m) !} p^{m}(1-p)^{n-m}
$$

The probability of the event happening $m$ or more times is:

$$
P(m+, n, p)=\sum_{i=m}^{n} P(i, n, p)
$$

Thus $P(m, n, p(v-i))$ is the probability that $m$ or more occurrences of patterns for $i$ will occur with a verb which is not a member of $i$, given $n$ occurrences of that verb. Setting a threshold of less than or equal to 0.05 yields a $95 \%$ or better confidence that a high enough proportion of patterns for $i$ have been observed for the verb to be in class $i^{3}$.

\subsection{Discussion}

Our approach to acquiring subcategorization classes is predicated on the following assumptions:

- most sentences will not allow the application of all possible rules of English complementation;

- some sentences will be unambiguous even given the indeterminacy of the grammar ${ }^{4}$;

\footnotetext{
${ }^{3}$ Brent (1993:249-253) provides a detailed explanation and justification for the use of this measure.

${ }^{4}$ In fact, $5 \%$ of sentences in Susanne are assigned only a single analysis by the grammar.
}

- many incorrect analyses will yield patterns which are unclassifiable, and are thus filtered out;

- arguments of a specific verb will occur with greater frequency than adjuncts (in potential argument positions);

- the patternset generator will incorrectly output patterns for certain classes more often than others; and

- even a highest ranked pattern for $i$ is only a probabilistic cue for membership of $i$, so membership should only be inferred if there are enough occurrences of patterns for $i$ in the data to outweigh the error probability for $i$.

This simple automated, hybrid linguistic/statistical approach contrasts with the manual linguistic analysis of the COMLEX Syntax lexicographers (Meyers et al., 1994), who propose five criteria and five heuristics for argument-hood and six criteria and two heuristics for adjunct-hood, culled mostly from the linguistics literature. Many of these are not exploitable automatically because they rest on semantic judgements which cannot (yet) be made automatically: for example, optional arguments are often 'understood' or implied if missing. Others are syntactic tests involving diathesis alternation possibilities (e.g. passive, dative movement, Levin (1993)) which require recognition that the 'same' argument, defined usually by semantic class / thematic role, is occurring across argument positions. We hope to exploit this information where possible at a later stage in the development of our approach. However, recognizing same/similar arguments requires considerable quantities of lexical data or the ability to back-off to lexical semantic classes. At the moment, we exploit linguistic information about the syntactic type, obligatoriness and position of arguments, as well as the set of possible subcategorization classes, and combine this with statistical inference based on the probability of class membership and the frequency and reliability of patterns for classes.

\section{Experimental Evaluation}

\subsection{Lexicon Evaluation - Method}

In order to test the accuracy of our system (as developed so far) and to provide empirical feedback for further development, we took the Susanne, SEC (Taylor \& Knowles, 1988) and LOB corpora (Garside et al., 1987) - a total of 1.2 million words-and extracted all sentences containing an occurrence of one of fourteen verbs, up to a maximum of 1000 citations of each. These verbs, listed in Figure 2, were chosen at random, subject to the constraint that they exhibited multiple complementation patterns. The sentences containing these verbs were tagged and parsed automatically, and the extractor, classifier and evaluator were applied to the resulting 
successful analyses. The citations from which entries were derived totaled approximately $70 \mathrm{~K}$ words.

The results were evaluated against a merged entry for these verbs from the ANLT and COMLEX Syntax dictionaries, and also against a manual analysis of the corpus data for seven of the verbs. The process of evaluating the performance of the system relative to the dictionaries could, in principle, be reduced to an automated report of type precision (percentage of correct subcategorization classes to all classes found) and recall (percentage of correct classes found in the dictionary entry). However, since there are disagreements between the dictionaries and there are classes found in the corpus data that are not contained in either dictionary, we report results relative both to a manually merged entry from ANLT and COMLEX, and also, for seven of the verbs, to a manual analysis of the actual corpus data. The latter analysis is necessary because precision and recall measures against the merged entry will still tend to yield inaccurate results as the system cannot acquire classes not exemplified in the data, and may acquire classes incorrectly absent from the dictionaries.

We illustrate these problems with reference to seem, where there is overlap, but not agreement between the COMLEX and ANLT entries. Thus, both predict that seem will occur with a sentential complement and dummy subject, but only ANLT predicts the possibility of a ' $w h$ ' complement and only COMLEX predicts the (optional) presence of a PP[to] argument with the sentential complement. One ANLT entry covers two COMLEX entries given the different treatment of the relevant complements but the classifier keeps them distinct. The corpus data for seem contains examples of further classes which we judge valid, in which seem can take a $\mathrm{PP}[\mathrm{to}]$ and infinitive complement, as in he seems to me to be insane, and a passive participle, as in he seemed depressed. This comparison illustrates the problem of errors of omission common to computational lexicons constructed manually and also from machine-readable dictionaries. All classes for seem are exemplified in the corpus data, but for ask, for example, eight classes (out of a possible 27 in the merged entry) are not present, so comparison only to the merged entry would give an unreasonably low estimate of recall.

\subsection{Lexicon Evaluation - Results}

Figure 2 gives the raw results for the merged entries and corpus analysis on each verb. It shows the number of true positives (TP), correct classes proposed by our system, false positives (FP), incorrect classes proposed by our system, and false negatives $(\mathrm{FN})$, correct classes not proposed by our system, as judged against the merged entry, and, for seven of the verbs, against the corpus analysis. It also shows, in the final column, the number of sentences from which classes were extracted.

\begin{tabular}{||l|r|r||}
\hline & $\begin{array}{r}\text { Dictionary } \\
\text { (14 verbs) }\end{array}$ & $\begin{array}{r}\text { Corpus } \\
(7 \text { verbs) }\end{array}$ \\
\hline Precision & $65.7 \%$ & $76.6 \%$ \\
Recall & $35.5 \%$ & $43.4 \%$ \\
\hline
\end{tabular}

Figure 3: Type precision and recall

\begin{tabular}{||l|r||}
\hline & Ranking Accuracy \\
\hline ask & $75.0 \%$ \\
begin & $100.0 \%$ \\
believe & $66.7 \%$ \\
cause & $100.0 \%$ \\
give & $70.0 \%$ \\
seem & $75.0 \%$ \\
swing & $83.3 \%$ \\
\hline Mean & $81.4 \%$ \\
\hline
\end{tabular}

Figure 4: Ranking accuracy of classes

Figure 3 gives the type precision and recall of our system's recognition of subcategorization classes as evaluated against the merged dictionary entries (14 verbs) and against the manually analysed corpus data ( 7 verbs). The frequency distribution of the classes is highly skewed: for example for believe, there are 107 instances of the most common class in the corpus data, but only 6 instances in total of the least common four classes. More generally, for the manually analysed verbs, almost $60 \%$ of the false negatives have only one or two exemplars each in the corpus citations. None of them are returned by the system because the binomial filter always rejects classes hypothesised on the basis of such little evidence.

In Figure 4 we estimate the accuracy with which our system ranks true positive classes against the correct ranking for the seven verbs whose corpus input was manually analysed. We compute this measure by calculating the percentage of pairs of classes at positions $(n, m)$ s.t. $n<m$ in the system ranking that are ordered the same in the correct ranking. This gives us an estimate of the accuracy of the relative frequencies of classes output by the system.

For each of the seven verbs for which we undertook a corpus analysis, we calculate the token recall of our system as the percentage (over all exemplars) of true positives in the corpus. This gives us an estimate of the parsing performance that would result from providing a parser with entries built using the system, shown in Figure 5.

Further evaluation of the results for these seven verbs reveals that the filtering phase is the weak link in the system. There are only 13 true negatives which the system failed to propose, each exemplified in the data by a mean of 4.5 examples. On the other hand, there are 67 false negatives supported by an estimated mean of 7.1 examples which should, ide- 


\begin{tabular}{||l||r|r|r||r|r|r||r||}
\hline \multicolumn{1}{||c||}{} & \multicolumn{3}{c||}{ Merged Entry } & \multicolumn{3}{c||}{ Corpus Data } & \multicolumn{2}{c||}{ No. of } \\
& TP & FP & FN & TP & FP & FN & Sentences \\
\hline ask & 9 & 0 & 18 & 9 & 0 & 10 & 390 \\
begin & 4 & 1 & 7 & 4 & 1 & 7 & 311 \\
believe & 4 & 4 & 11 & 4 & 4 & 8 & 230 \\
cause & 2 & 3 & 6 & 2 & 3 & 5 & 95 \\
expect & 6 & 5 & 3 & - & - & - & 223 \\
find & 5 & 7 & 15 & - & - & - & 645 \\
give & 5 & 2 & 11 & 5 & 2 & 5 & 639 \\
help & 6 & 3 & 8 & - & - & - & 223 \\
like & 3 & 2 & 7 & - & - & - & 228 \\
move & 4 & 3 & 9 & - & - & - & 217 \\
produce & 2 & 1 & 3 & - & - & - & 152 \\
provide & 3 & 2 & 6 & - & - & - & 217 \\
seem & 8 & 1 & 4 & 8 & 1 & 4 & 534 \\
swing & 4 & 0 & 10 & 4 & 0 & 8 & 45 \\
\hline Totals & 65 & 34 & 118 & 36 & 11 & 47 & 4149 \\
\hline
\end{tabular}

Figure 2: Raw results for test of 14 verbs

\begin{tabular}{||l|r||}
\hline & Token Recall \\
\hline ask & $78.5 \%$ \\
begin & $73.8 \%$ \\
believe & $34.5 \%$ \\
cause & $92.1 \%$ \\
give & $92.2 \%$ \\
seem & $84.7 \%$ \\
swing & $39.2 \%$ \\
\hline Mean & $80.9 \%$ \\
\hline
\end{tabular}

Figure 5: Token recall

ally, have been accepted by the filter, and 11 false positives which should have been rejected. The performance of the filter for classes with less than 10 exemplars is around chance, and a simple heuristic of accepting all classes with more than 10 exemplars would have produced broadly similar results for these verbs. The filter may well be performing poorly because the probability of generating a subcategorization class for a given verb is often lower than the error probability for that class.

\subsection{Parsing Evaluation}

In addition to evaluating the acquired subcategorization information against existing lexical resources, we have also evaluated the information in the context of an actual parsing system. In particular we wanted to establish whether the subcategorization frequency information for individual verbs could be used to improve the accuracy of a parser that uses statistical techniques to rank analyses.

The experiment used the same probabilistic parser and tag sequence grammar as are present in the acquisition system (see references above) - although the experiment does not in any way rely on the

\begin{tabular}{||l|rrr||}
\hline & $\begin{array}{c}\text { Mean } \\
\text { crossings }\end{array}$ & Recall & Precision \\
\hline 'Baseline' & 1.00 & $70.7 \%$ & $72.3 \%$ \\
Lexicalised & 0.93 & $71.4 \%$ & $72.9 \%$ \\
\hline
\end{tabular}

Figure 6: GEIG evaluation metrics for parser against Susanne bracketings

parsers or grammars being the same. We randomly selected a test set of 250 in-coverage sentences (of lengths 3-56 tokens, mean 18.2) from the Susanne treebank, retagged with possibly multiple tags per word, and measured the 'baseline' accuracy of the unlexicalized parser on the sentences using the now standard PARSEVAL/GEIG evaluation metrics of mean crossing brackets per sentence and (unlabelled) bracket recall and precision (e.g. Grishman et al., 1992); see figure $6^{5}$. Next, we collected all words in the test corpus tagged as possibly being verbs (giving a total of 356 distinct lemmas) and retrieved all citations of them in the LOB corpus, plus Susanne with the 250 test sentences excluded. We acquired subcategorization and associated frequency information from the citations, in the process successfully parsing $380 \mathrm{~K}$ words. We then parsed the test set, with each verb subcategorization possibility weighted by its raw frequency score, and using the naive add-one smoothing technique to allow for omitted possibilities. The GEIG measures for the lexicalized parser show a $7 \%$ improvement in the crossing bracket score (figure 6). Over the existing test corpus this is not statisti-

\footnotetext{
${ }^{5}$ Carroll \& Briscoe (1996) use the same test set, although the baseline results reported here differ slightly due to differences in the mapping from parse trees to Susanne-compatible bracketings.
} 
cally significant at the $95 \%$ level (paired $t$-test, 1.21 , $249 d f, p=0.11$ ) -although if the pattern of differences were maintained over a larger test set of 470 sentences it would be significant. We expect that a more sophisticated smoothing technique, a larger acquisition corpus, and extensions to the system to deal with nominal and adjectival predicates would improve accuracy still further. Nevertheless, this experiment demonstrates that lexicalizing a grammar/parser with subcategorization frequencies can appreciably improve the accuracy of parse ranking.

\section{Related Work}

Brent's (1993) approach to acquiring subcategorization is based on a philosophy of only exploiting unambiguous and determinate information in unanalysed corpora. He defines a number of lexical patterns (mostly involving closed class items, such as pronouns) which reliably cue one of five subcategorization classes. Brent does not report comprehensive results, but for one class, sentential complement verbs, he achieves $96 \%$ precision and $76 \%$ recall at classifying individual tokens of 63 distinct verbs as exemplars or non-exemplars of this class. He does not attempt to rank different classes for a given verb.

Ushioda et al. (1993) utilise a PoS tagged corpus and finite-state NP parser to recognize and calculate the relative frequency of six subcategorization classes. They report an accuracy rate of $83 \%$ (254 errors) at classifying 1565 classifiable tokens of 33 distinct verbs in running text and suggest that incorrect noun phrase boundary detection accounts for the majority of errors. They report that for 32 verbs their system correctly predicts the most frequent class, and for 30 verbs it correctly predicts the second most frequent class, if there was one. Our system rankings include all classes for each verb, from a total of 160 classes, and average $81.4 \%$ correct.

Manning (1993) conducts a larger experiment, also using a PoS tagged corpus and a finite-state NP parser, attempting to recognize sixteen distinct complementation patterns. He reports that for a test sample of 200 tokens of 40 verbs in running text, the acquired subcategorization dictionary listed the appropriate entry for 163 cases, giving a token recall of $82 \%$ (as compared with $80.9 \%$ in our experiment). He also reports a comparison of acquired entries for the verbs to the entries given in the Oxford Advanced Learner's Dictionary of Current English (Hornby, 1989 ) on which his system achieves a precision of $90 \%$ and a recall of $43 \%$. His system averages 3.48 subentries (maximum 10) -less then half the number produced in our experiment. It is not clear what level of evidence the performance of Manning's system is based on, but the system was applied to 4.1 million words of text (c.f. our 1.2 million words) and the verbs are all common, so it is likely that considerably more exemplars of each verb were available.

\section{Conclusions and Further Work}

The experiment and comparison reported above suggests that our more comprehensive subcategorization class extractor is able both to assign classes to individual verbal predicates and also to rank them according to relative frequency with comparable accuracy to extant systems. We have also demonstrated that a subcategorization dictionary built with the system can improve the accuracy of a probabilistic parser by an appreciable amount.

The system we have developed is straightforwardly extensible to nominal and adjectival predicates; the existing grammar distinguishes nominal and adjectival arguments from adjuncts structurally, so all that is required is extension of the classifier. Developing an analogous system for another language would be harder but not infeasible; similar taggers and parsers have been developed for a number of languages, but no extant subcategorization dictionaries exist to our knowledge, therefore the lexical statistics we utilize for statistical filtering would need to be estimated, perhaps using the technique described by Brent (1993). However, the entire approach to filtering needs improvement, as evaluation of our results demonstrates that it is the weakest link in our current system.

Our system needs further refinement to narrow some subcategorization classes, for example, to choose between differing control options with predicative complements. It also needs supplementing with information about diathesis alternation possibilities (e.g. Levin, 1993) and semantic selection preferences on argument heads. Grishman \& Sterling (1992), Poznanski \& Sanfilippo (1993), Resnik (1993), Ribas (1994) and others have shown that it is possible to acquire selection preferences from (partially) parsed data. Our system already gathers head lemmas in patterns, so any of these approaches could be applied, in principle. In future work, we intend to extend the system in this direction. The ability to recognize that argument slots of different subcategorization classes for the same predicate share semantic restrictions/preferences would assist recognition that the predicate undergoes specific alternations, this in turn assisting inferences about control, equi and raising (e.g. Boguraev \& Briscoe, 1987).

\section{References}

Boguraev, B. \& Briscoe, E. 1987. Large lexicons for natural language processing: utilising the grammar coding system of the Longman Dictionary of Contemporary English. Computational Linguistics 13.4: 219-240.

Boguraev, B. \& Briscoe, E. 1989. Introduction. In Boguraev, B. \& Briscoe, E. eds. Computational Lexicography for Natural Language Processing. Longman, London: 1-40.

Boguraev, B., Briscoe, E., Carroll, J., Carter, D. 
\& Grover, C. 1987. The derivation of a grammatically-indexed lexicon from the Longman Dictionary of Contemporary English. In Proceedings of the 25th Annual Meeting of the Association for Computational Linguistics, Stanford, CA. 193-200.

Brent, M. 1991. Automatic acquisition of subcategorization frames from untagged text. In Proceedings of the 29th Annual Meeting of the Association for Computational Linguistics, Berkeley, CA. 209-214.

Brent, M. 1993. From grammar to lexicon: unsupervised learning of lexical syntax. Computational Linguistics 19.3: 243-262.

Briscoe, E. \& Carroll, J. 1993. Generalised probabilistic LR parsing for unification-based grammars. Computational Linguistics 19.1: 25-60.

Briscoe, E. \& Carroll, J. 1994. Parsing (with) punctuation. Rank Xerox Research Centre, Grenoble, MLTT-TR-007.

Briscoe, E. \& Carroll, J. 1995. Developing and evaluating a probabilistic LR parser of part-of-speech and punctuation labels. In Proceedings of the 4 th $A C L / S I G P A R S E$ International Workshop on Parsing Technologies, Prague, Czech Republic. 48-58.

Carroll, J. 1993. Practical unification-based parsing of natural language. Cambridge University Computer Laboratory, TR-224.

Carroll, J. 1994. Relating complexity to practical performance in parsing with wide-coverage unification grammars. In Proceedings of the 32nd Annual Meeting of the Association for Computational Linguistics, NMSU, Las Cruces, NM. 287-294.

Carroll, J. \& Briscoe, E. 1996. Apportioning development effort in a probabilistic LR parsing system through evaluation. In Proceedings of the $A C L$ SIGDAT Conference on Empirical Methods in Natural Language Processing, University of Pensylvania, Philadelphia, PA. 92-100.

Carroll, J. \& Grover, C. 1989. The derivation of a large computational lexicon for English from LDOCE. In Boguraev, B. and Briscoe, E. eds. Computational Lexicography for Natural Language Processing. Longman, London: 117-134.

Cunningham, H., Gaizauskas, R. \& Wilks, Y. 1995. A general architecture for text engineering (GATE) - a new approach to language $R E D$. Research memo CS-95-21, Department of Computer Science, University of Sheffield, UK.

de Marcken, C. 1990. Parsing the LOB corpus. In Proceedings of the 28th Annual Meeting of the Association for Computational Linguistics, Pittsburgh, PA. 243-251.

Elworthy, D. 1994. Does Baum-Welch re-estimation help taggers?. In Proceedings of the 4th Conf. $A p$ plied NLP, Stuttgart, Germany.

Garside, R., Leech, G. \& Sampson, G. 1987. The computational analysis of English: $A$ corpus-based approach. Longman, London.

Grishman, R., Macleod, C. \& Meyers, A. 1994. Comlex syntax: building a computational lexi- con. In Proceedings of the International Conference on Computational Linguistics, COLING-94, Kyoto, Japan. 268-272

Grishman, R., Macleod, C. \& Sterling, J. 1992. Evaluating parsing strategies using standardized parse files. In Proceedings of the 3rd $A C L$ Conference on Applied Natural Language Processing, Trento, Italy. 156-161.

Grishman, R. \& Sterling, J. 1992. Acquisition of selectional patterns. In Proceedings of the International Conference on Computational Linguistics, COLING-92, Nantes, France. 658-664.

Jackendoff, R. 1977. X-bar syntax. MIT Press; Cambridge, MA.

Jensen, K. 1991. A broad-coverage natural language analysis system. In M. Tomita eds. Current Issues in Parsing Technology. Kluwer, Dordrecht.

Levin, B. 1993. Towards a lexical organization of English verbs. Chicago University Press, Chicago.

Manning, C. 1993. Automatic acquisition of a large subcategorisation dictionary from corpora. In Proceedings of the 31st Annual Meeting of the Association for Computational Linguistics, Columbus, Ohio. 235-242.

Meyers, A., Macleod, C. \& Grishman, R. 1994. Standardization of the complement adjunct distinction. New York University, Ms.

Nunberg, G. 1990. The linguistics of punctuation. CSLI Lecture Notes 18, Stanford, CA.

Poznanski, V. \& Sanfilippo, A. 1993. Detecting dependencies between semantic verb subclasses and subcategorization frames in text corpora. In Proceedings of the SIGLEX ACL Workshop on the Acquisition of Lexical Knowledge from Text, Boguraev, B. \& Pustejovsky, J. eds.

Resnik, P. 1993. Selection and information: a classbased approach to lexical relationships. University of Pennsylvania, CIS Dept, PhD thesis.

Ribas, P. 1994. An experiment on learning appropriate selection restrictions from a parsed corpus. In Proceedings of the International Conference on Computational Linguistics, COLING-94, Kyoto, Japan.

Sampson, G. 1995. English for the computer. Oxford, UK: Oxford University Press.

Schabes, Y. 1992. Stochastic lexicalized tree adjoining grammars. In Proceedings of the International Conference on Computational Linguistics, COLING-92, Nantes, France. 426-432.

Taylor, L. \& Knowles, G. 1988. Manual of information to accompany the $S E C$ corpus: the machinereadable corpus of spoken English. University of Lancaster, UK, Ms.

Ushioda, A., Evans, D., Gibson, T. \& Waibel, A. 1993. The automatic acquisition of frequencies of verb subcategorization frames from tagged corpora. In Boguraev, B. \& Pustejovsky, J. eds. SIGLEX $A C L$ Workshop on the Acquisition of Lexical Knowledge from Text. Columbus, Ohio: 95-106. 